\title{
Um Estudo de Caso de Acesso à Opinião do Cidadão no Processo de Construção e Implementação de um Projeto de Cidades Inteligentes em Rio das Ostras
}

\author{
Flavia Bernardini ${ }^{1}$ \\ ${ }^{1}$ Departamento de Computação - Instituto de Ciência e Tecnologia - Universidade \\ Federal Fluminense (UFF) \\ Campus de Rio das Ostras - Rio das Ostras - RJ - Brazil \\ fcbernardini@id.uff.br
}

\begin{abstract}
Resumo. Atualmente, diversos projetos de Cidades Inteligentes são implementados nas cidades. No entanto, poucas cidades acessam explicitamente a opinião de sua população para verificar as ações a serem executadas. $O$ objetivo deste trabalho é apresentar um estudo de caso, realizado em Rio das Ostras/RJ, para acessar a opinião da população quanto a ações a serem realizadas no contexto do seu projeto de Cidades Inteligentes.
\end{abstract}

\begin{abstract}
Nowadays, diverse projects in Smart Cities are implemented in cities. However, few cities explicitly assess its population opinion to verify actions that should be executed. The purpose of this work is to present a case study, conducted in Rio das Ostra/RJ, to assess population opinion related to actions to be executed in its Smart City project context.
\end{abstract}

\section{Introdução}

Diversos projetos para implantação de soluções em Cidades Inteligentes (CIs) têm sido executados ao redor do mundo. Em muitos deles, é possível observar que não é clara a participação popular na tomada de decisão quanto a que aspectos devem ser tratados. A participação popular pode ocorrer por diversos mecanismos, mas só é efetiva se a população tiver ao menos acesso a informações. Segundo [ALÓ 2009], o primeiro degrau da transparência está na acessibilidade à informação. Em cidades, isso se obtém inicialmente por meio da disponibilização de dados abertos em portais públicos. No entanto, em diversos municípios brasileiros, incluindo Rio das Ostras, tal portal não existe. Outros mecanismos de acesso podem ser utilizados, como sessões públicas, questionários virtuais, living labs, dentre outros. A dificuldade de quaisquer desses mecanismos é permitir a ampla participação da população em uma cidade. No entanto, iniciativas pilotos também são importantes.

Para promover as diversas práticas de transparência, bem como diversas outras práticas consideradas importantes, do ponto de vista das definições de CIs apresentadas na literatura, foi criada no Brasil a Rede Brasileira de Cidades Inteligentes e Humanas $(\mathrm{RBCIH})^{1}$ em 2014, e Rio das Ostras faz parte dessa rede. A intenção do governo

\footnotetext{
${ }^{1}$ Mais informações sobre a Rede Brasileira de Cidades Inteligentes e Humanas podem ser encontradas em http://www.redebrasileira.org/.
} 
municipal foi executar um projeto no município com soluções que vão ao encontro do conceito de CI definido pela RBCIH. Para permitir que a população pudesse se manifestar quanto ao projeto, um grupo de pessoas, capitaneado pela Secretaria de Ciência e Tecnologia (SECTI) do município, e formado por representantes do governo municipal, da Instituição de Ciência e Tecnologia (ICT) local (a UFF), da iniciativa privada local, e da população em geral, planejaram um evento para lançamento do projeto, o III Encontro da Cidade com a Ciência (III ENCIC), em junho de 2016. O evento também objetivou apresentar a definição de CI da RBCIH, e criar um espaço público para discussão e acesso à população quanto suas intenções.

O objetivo deste trabalho é apresentar um estudo de caso que avalia o mecanismo de acesso às informações da população para definição dos rumos do projeto de CIs para o município de Rio das Ostras, utilizado no evento. Para embasar a discussão, são apresentados, na Seção 2, conceitos e definições de CIs nos quais esse trabalho se embasa e conceitos de transparência utilizados; e na Seção 3, uma revisão da literatura quanto a métodos utilizados em diversas cidades do mundo, para permitir o acesso à informação, fornecida pelos cidadãos. Para o desenvolvimento do estudo de caso, descrito na Seção 4, é apresentado como o evento foi planejado e executado, e ao final é apresentada uma análise dos resultados obtidos. Na Seção 5, são apresentadas as conclusões deste trabalho e trabalhos futuros.

\section{Conceitos e Definições}

\subsection{Cidades Inteligentes}

Historicamente, o termo "Cidades Inteligentes" surgiu pela primeira vez em 1997, mas se tornou popular somente em 2010. Para [COCCHIA 2014], uma cidade é dita digital quando segue políticas digitais para suprir serviços eletrônicos aos cidadãos por meio do uso das Tecnologias da Informação e Comunicação (TICs); é dita inteligente quando segue estratégias sustentáveis pelo uso sustentável e com inovação de seus próprios recursos naturais; e é dita inteligente baseada no digital quando segue estratégias sustentáveis usando tecnologias aplicadas a cidades digitais. Já segundo [GIFFINGER e GUDRUN 2010], "uma cidade é inteligente quando seus investimentos em capital humano e social, em transporte urbano e infraestrutura de TIC alimentam o desenvolvimento econômico sustentável e uma melhor qualidade de vida, com sábio gerenciamento de recursos naturais, através do governo participativo". A partir dessa definição, os autores também propuseram um modelo de referência para avaliação de CIs de médio porte da União Européia. Nesse modelo são consideradas seis principais dimensões para englobar as iniciativas em CIs nas cidades: economia inteligente, mobilidade inteligente, ambiente inteligente, pessoas inteligentes, vida inteligente e governança inteligente. Diversos outros autores também usam essa definição e abordagem de divisão de CIs em seis dimensões.

No entanto, em [DAMERI e ROSENTHAL-SABROUX 2014], os autores afirmam que indicar alguma iniciativa como pertencente a uma única dimensão muitas vezes é difícil, devido a possíveis intersecções entre as dimensões. Por exemplo, um novo sistema de transporte público baseado em baixa emissão de carbono impacta tanto em mobilidade quanto em ambiente inteligente. Assim, em [COCCHIA 2014] é apresentada uma evolução no conceito de CIs. São apresentados quatro componentes 
centrais, que devem ser considerados em uma cidade para ser dita mais inteligente terreno, infraestrutura, pessoas e governo. Esses componentes devem ser abordados sob três aspectos: (i) eficácia, relacionada à capacidade da cidade de suprir efetivamente serviços públicos e privados para os diferentes órgãos da sociedade (cidadãos, companhias e organizações sem fins lucrativos); e para diferentes categorias de cidadãos (estudantes, trabalhadores, idosos, etc); (ii) considerações ambientais, relacionadas à capacidade de medir o impacto do crescimento da cidade na qualidade do ambiente nas áreas urbanas; e (iii) inovação, que sinaliza que uma CI deve usar todas as mais atuais tecnologias para melhorar a qualidade dos seus componentes principais, para entregar melhores serviços e reduzir os impactos ambientais. É importante observar que o trabalho ainda aponta que, em eficácia, deve ser incluído o papel subjetivo de cada parte interessada na definição de inteligente, ou seja, a cidade não é inteligente por si mesma, mas sim deve ser criado valor público para os cidadãos. Também, no aspecto Inovação, deve-se considerar que a tecnologia é um aspecto central da CI, usada no serviço das iniciativas inteligentes para a qualidade de vida na cidade.

Assim, a partir desses conceitos e das características regionais descritas, é necessário escolher uma definição de CI para especificar sua própria visão de estratégia de $\mathrm{CI}$ e construir seu próprio modelo de CI, para que o valor agregado aos cidadãos seja discutido e definido. Em [LOMBARDI et al. 2011] foi definido um framework baseado no modelo de Hélice Tríplice, para conectar todos os projetos e iniciativas ligados ao conceito de CIs. O modelo de Hélice Tríplice emergiu como um quadro de referência para a análise dos sistemas de inovação baseada no conhecimento, e relaciona as relações múltiplas e recíprocas entre os três principais agentes no processo de criação de conhecimento e capitalização: universidade, indústria e governo. Os autores evoluem o modelo de hélice tríplice para embasar o desenvolvimento de CIs em termos de seus papéis tradicionais e contemporâneos, inicialmente como geradoras de capital intelectual, criadoras de riqueza e reguladoras de normas, com participação da universidade, indústria e governo, respectivamente; para, logo em seguida, definir como cidades que usam tais atributos devem ser inteligentes no apoio ao aprendizado social, capacidades empresariais baseadas no mercado e habilidades de transferência de conhecimentos, necessários para satisfazer as exigências dos seus sistemas de inovação regionais. Uma evolução do conceito de tríplice hélice para inovação em cidades é o modelo de hélice quádrupla [CARAYANNIS e CAMPBELL 2009], e referem-se a novas configurações sociais para a organização da inovação onde o governo, indústria, academia e participantes civis trabalham juntos para cocriar o futuro e conduzir mudanças estruturais muito além do escopo do que qualquer organização ou pessoa poderia fazer individualmente. Uma das maneiras de garantir a implementação do modelo de hélice quádrupla é por meio de living labs, ou laboratórios vivos. Living labs são apontados no Horizonte 2020 - Programa-Quadro de Investigação e Inovação [EUROPEAN COMMISSION 2011] como o mecanismo que pode abranger dimensões sociais e tecnológicas, simultaneamente, em PPPPs - Parcerias-Pessoais-PúblicoPrivadas. Em [SILVA e BITTENCOURT 2015], os autores revisitam conceitos de living lab, e apresentam uma sugestão de definição, condensando diversas definições da literatura. Living labs podem ser percebidos como uma arena para a inovação, uma estrutura e uma comunidade de longo prazo relacionada a um único projeto, onde experiências, rotinas e condições são construídos para transformar ideias em inovações. 


\subsection{Acessando os Interesses da População nas Cidades}

Diversos trabalhos são encontrados na internet para acesso à opinião e participação de cidadãos para os mais diversos temas em CIs. Para este trabalho, foram selecionados, dentre os diversos trabalhos encontrados, apenas três trabalhos que discutem métodos utilizados para acessar a opinião da população das cidades, tanto para geração de ideias quanto para avaliação de soluções, que mais se aproximam do trabalho desenvolvido descrito neste artigo.

Em [BAKICI, ALMIRALL e WAREHAM 2013] é apresentado um estudo de caso de CI em Barcelona. O objetivo do estudo é ilustrar uma CI em um contexto real enquanto examina sua transformação. $\mathrm{O}$ método utilizado para o estudo envolveu entrevistas e análises de sítios na web. No trabalho, os autores afirmam que Barcelona é considerada um caso de sucesso em CIs na Europa, por possuir diversas iniciativas no setor industrial, denominadas distritos inteligentes. Esses distritos possuem uma economia intensamente baseada no conhecimento, o que cria um espaço de relacionamentos sociais entre companhias, instituições, prefeituras e cidadãos, e essa interação leva a uma sociedade do conhecimento. Barcelona também é importante no contexto de CIs por sua tendência em políticas e reformas públicas urbanas. Por esses motivos que o acesso à população, quanto à iniciativa de CIs, pôde trazer luz às políticas públicas urbanas da cidade, auxiliando nas direções futuras. Para isso, Barcelona usa o conceito de living lab nos distritos inteligentes, que promovem a inovação nas companhias. Ainda, a prefeitura da cidade também tem um projeto de dados abertos para disponibilizar as informações públicas ao cidadão, incluindo indicadores de território, população e gestão pública, ambiente urbano, dentre outros. Por fim, o gerenciamento da iniciativa de CIs em Barcelona é realizado por várias organizações e departamentos, além do departamento municipal de mobilidade, do instituto municipal de Tecnologia da Informação (TI) e de uma agência de promoção econômica.

Em [MECHANT et al. 2012] é apresentada a importância de uma abordagem orientada na concepção e desenvolvimento de serviços e aplicações inovadoras para cidades. São relatados no trabalho dois estudos de caso realizados em Ghent, na Bélgica, apresentando como diferentes métodos e estratégias foram utilizados na geração e avaliação de conceitos e ideias inovadoras em CIs. Para cada estudo de caso, é apresentado (i) o contexto e a estratégia da política utilizada; (ii) os problemas encontrados e soluções alcançadas; e (iii) as abordagens de e-deliberação utilizadas. $\mathrm{O}$ termo "e-deliberação" está relacionado à adoção de práticas para sustentar a democracia participativa. O artigo tem como foco o uso de ferramentas para e-deliberação para gerar ideias em duas diferentes áreas de aplicação em CIs. Para geração de ideias da primeira aplicação, foi utilizada a técnica de brainstorming e um questionário online para cidadãos responderam. A avaliação das ideias também foi realizada em ferramenta online. Os autores avaliaram a eficácia e o impacto das abordagens, concluindo que tanto ambas as estratégias de geração de ideias como a de avaliação funcionam. Para eles, a importância do envolvimento do cidadão desde as primeiras fases do processo adotado foi indispensável por levar a maiores taxas de adoção das soluções desenvolvidas por parte da população.

Para [SCHUURMAN et al. 2012], o conceito inteligente para uma cidade deve ser mais centrada no usuário do que outros conceitos mais tecnológicos, como o 
conceito de cidade digital. Assim, os autores apresentam princípios e diferentes manifestações de crowdsourcing por meio de (i) uma discussão dos pontos fortes e fracos do crowdsourcing para geração e seleção de ideias para inovação em cidades; e (ii) um estudo de caso de geração e seleção de ideias para inovação urbana por meio de uma plataforma on-line. A solução de crowdsourcing é comparada a uma geração realizada por especialistas externos. A comparação de ambos indica que usando a multidão como gerador e seletor de ideias inovadoras produz uma longa lista com altos benefícios para o usuário. Porém, as ideias não parecem ser muito inovadoras. Assim, os autores concluem que crowdsourcing aparenta ser uma ferramenta útil e eficaz no contexto de inovação inteligente das cidades, mas deve ser utilizada e combinada com outras abordagens de envolvimento do cidadão, como living labs. Isso reforça o uso de iniciativas que unem a população presencialmente no processo de construção e implementação de um projeto de CI.

\section{Um Estudo de Caso - Acesso à Opinião em Rio das Ostras}

Em [TERÁN, KASHINA e MEIER 2016] é apresentado um modelo de maturidade para Cidades Cognitivas, e os autores destacam a importância da interação entre a administração pública e os cidadãos. Assim, utilizam um framework de governo eletrônico (e-gov) para garantir o empoderamento eletrônico por parte da população. Nesse framework, a população somente opina nos projetos após o acesso via portais eletrônicos. No entanto, como apontado por [SCHUURMAN et al. 2012], a participação também pode se dar na evolução do projeto de CI, envolvendo todos os representantes de diversos setores interessados em uma cidade. Isso vai ao encontro do que foi apontado no modelo de Hélice Quádrupla, previamente apresentado. Assim, o sucesso de um projeto de CI em uma cidade depende da participação do cidadão, disponibilizando sua percepção do que é relevante. No caso do Brasil, a RBCIH aponta o conceito de CI para as cidades. Porém, não deixa claro como acessar a opinião do cidadão quanto ao valor agregado do projeto de CI para uma cidade. Um dos objetivos, portanto, do III ENCIC, em Rio das Ostras, foi levar aos cidadãos o conceito de CI da RBCIH, que amplia os conceitos apresentados na Seção 2.1, para tornar explícita a questão "Humana" de um projeto de CI para uma cidade, além de acessar a opinião do cidadão Riostrense quanto ao projeto e às necessidades da cidade.

\subsection{Descrição do evento para coleta de opinião dos cidadãos}

Antes do evento, foram realizadas diversas reuniões, com diversos integrantes da sociedade, para apresentar conceitos de CIs e possibilidades de inovação e melhoria para a cidade, e criar um espaço de discussão e amadurecimento das necessidades do município pela população. Para as reuniões, foram convidados representantes de diversos setores e associações de moradores. Após as reuniões, foi identificada a necessidade de criação de uma comissão para planejar o evento, em relação (i) à abordagem de lançamento do projeto para o município; (ii) ao levantamento de instituições e organizações que necessitariam ser convidadas; e (iii) ao desenvolvimento do método para coleta das opiniões dos participantes do evento. A comissão foi composta por membros da SECTI do município, professores do ICT local, representantes do comércio local e especialistas em inovação, seguindo os preceitos da abordagem de Hélice Quádrupla, apresentada anteriormente. Foi definido que, para 
permitir uma maior participação popular, seria interessante que o lançamento do projeto ocorreria em uma sexta-feira à noite, e que atividades envolvendo a população ocorreria no sábado.

Quanto ao item (i), a secretária da SECTI, juntamente com a comissão, decidiu que deveria haver a participação do presidente e vice-presidente da $\mathrm{RBCIH}$, além dos representantes do governo municipal, para fortalecer o lançamento do projeto. Quanto ao item (ii), o levantamento foi realizado pelos membros da comissão. Quanto ao item (iii), a comissão fez diversas reuniões, somente entre os membros, para alinhamento do método de acesso à população. Inicialmente, a equipe entendeu que, para as atividades com a população no sábado, seria interessante criar Grupos de Trabalho (GT) para discussão dos diversos temas inerentes a CIs. Foi definido ainda que os GTs iriam se reunir no sábado, na parte da manhã, em paralelo, e que, na parte da tarde, os resultados dos trabalhos dos GTs seriam consolidados numa reunião conjunta. Ao final das atividades no sábado, seria redigida uma carta de intenções para assinatura por parte do prefeito do município. Para agrupar os temas inerentes a CIs, foram utilizadas as dimensões definidas por [GIFFINGER e GUDRUN 2010] - economia, mobilidade, ambiente, pessoas, vida e governança inteligentes. Ainda, foi de entendimento comum que um GT seria formado para a dimensão economia, coordenado por um especialista em inovação; outro GT seria formado para englobar as dimensões governança, pessoa e vida inteligentes, coordenado por uma pesquisadora em cultura, uma pesquisadora em tecnologia, e a secretária de saúde do município; outro GT seria formado para a dimensão ambiente, para discutir, inclusive, questões de sustentabilidade, coordenado por um especialista em meio-ambiente; e outro GT para a dimensão mobilidade, coordenador por um pesquisador da área de logística e otimização. Participaram do evento 154 pessoas no lançamento do projeto, e 115 pessoas na execução dos GTs. Após reunião dos GTs, cada GT apontou as ações que devem ser realizadas no âmbito do projeto de CIs:

GT1 - Economia inteligente (8 ações): incentivo às escolas e aos professores para iniciativas em educação, para mudança de consciência em relação à transparência e o papel do cidadão nos processos transformadores da cidade; criação de canais de comunicação eficientes (p.ex., desenvolvimento de apps) para interagir com o cidadão, para amenizar a falta de comunicação; alteração das leis para agilizar os processos de criação de novas empresas; criação de um portal de transparência do município para tornar o acesso às informações eficiente e eficaz; criação de espaços para diálogo entre governo e população, com o objetivo de elaboração de políticas públicas; construção de um conselho de CI, fortalecendo e viabilizando as ações com o poder público; incentivo ao crescimento da economia local, por meio de ações que viabilizem startups e empreendedores, por meio de incubação, qualificação e desenvolvimento; continuação do trabalho desenvolvido no evento.

GT2 - Vida, Pessoa e Governança Inteligente (8 ações): o projeto deve ser iniciado com ações voltadas para transparência, com o governo e a governança como eixo central do projeto; ações em cultura e educação devem ter prioridade, pois as outras iniciativas só terão efetividade se o cidadão estiver consciente e apto para a participação; permissão de acesso à informação e à cultura, pois espaços de pronunciamento por parte e para a população é importante; execução de uma caravana cultural, por meio de linguagem lúdica, para mostrar a importância desses processos; desenvolvimento de 
procedimentos de base para mudar a cultura da corrupção no Brasil, pois os governantes precisam mudar a maneira de pensar, por meio da modificação da cultura em relação ao governo participativa; construção de um modelo de uma escola inteligente, pois escola é continuidade da casa da criança; ações que envolvem a cultura devem levar em consideração que é uma área antropológica e social, pois as atividades artísticas são expressões últimas da cultura; criação de um fórum, em um conselho de CI, com continuidade dos grupos de trabalho.

GT3 - Ambiente Inteligente (12 ações): construção de mecanismos de maior interação entre os poderes Executivo, Legislativo e Judiciário; construção de mecanismos de incentivo a atividades ligadas à criação de cooperativa(s) de catadores propiciando a geração de empregos com a valorização da mão-de-obra; criação de campanhas contínuas que incentivem práticas de separação de lixo; construção de mecanismos de incentivo à vinda e participação de empresas de gerenciamento de resíduos no município; maior uso de espaços públicos, como ambientes de discussão e reflexão em relação às questões ambientais, por meio de ações atrativas para esse fim; incentivo ao turismo local, de maneira que a população local também seja beneficiada com a prática turística; criação de ações efetivas de educação ambiental contínuas, envolvendo população e governabilidade, no conhecimento e reconhecimento da identidade ambiental local; efetivação de políticas públicas que levem em consideração as questões ambientais; viabilização de ações de responsabilidade social, econômica e ambiental, desenvolvidas para a sociedade, proporcionando o desenvolvimento de sistemas de TICs para gerenciamento e controle dos recursos naturais locais; criação de um núcleo regional de gerenciamento de resíduos sólidos, envolvendo diferentes esferas da sociedade nesse processo, por meio de parcerias entre órgãos públicos e privados, para o desenvolvimento de procedimentos e tecnologias para esse fim; criação de um aplicativo informativo sobre pontos de destinação de resíduos recicláveis, formas de separação, logística de recolhimento, divulgação de ações e iniciativas que visem a minimização da geração de resíduos; desenvolvimento de um sistema de informações geográficas, contemplando informações ambientais, econômicas e sociais.

GT4 - Mobilidade Inteligente (16 ações): construção de calçadas que permitam melhor mobilidade das pessoas; construção de aplicativos colaborativos para que os cidadãos possam ajudar a fiscalização das calçadas; construção de ciclovias em toda a cidade; construção de maneiras de educar o cidadão no seu comportamento no trânsito; ajuste do trânsito na principal rodovia do município, por meio de simulação computacional; uso de simulação computacional para estudos, relativos à construção de uma segunda via principal; adequação do transporte atual, baseados em transportes que apresentem qualidade adequada; integrações de linhas de transporte público; uso de semáforos inteligentes que se ajustem conforme o fluxo de veículos; uso de simulação computacional para melhorias no trânsito, considerando diferentes cenários; desenvolvimento de aplicativos para que o cidadão possa conhecer e fazer melhor uso do transporte público; uso de técnicas computacionais para ajustar linhas de transporte público; uso de marcações para formalizar estacionamento de veículos; proposta e implementação de formas de ajudar o cidadão a encontrar vagas; incentivo à pesquisa acadêmica em parceria com o governo municipal para busca de soluções de mobilidade urbana; planejamento e melhoria da mobilidade urbana para longo prazo, envolvendo setor público, academia, iniciativa privada e cidadão. 


\subsection{Análise do Resultados Obtidos no Evento}

Após a realização do evento, foi construído um questionário na web para que os participantes respondessem e pudessem comentar sobre o evento, que foi respondido por 82 pessoas. Foi observado que: os participantes gostaram da programação do evento (86\% dos respondentes); e acharam que (i) a organização do evento foi boa ou excelente (76,8\%); (ii) a divulgação do evento foi de média a excelente $(90,6 \%)$; (iii) o conhecimento dos palestrantes foi de nível médio a excelente (100\%); e (iv) as instalações eram boas ou excelentes $(80,9 \%)$. O grau de satisfação geral do evento foi de médio a excelente $(100 \%)$. Os respondentes também podiam deixar comentários e opiniões ao final do questionário, e um ponto criticado por mais de um participante foi falta de pontualidade do início das atividades do evento. Um respondente disse que "Todo conhecimento oferecido foi a partir de uma visão elitista. Precisava de um diagnóstico a partir da opinião da população. Não adianta fazer análise acadêmica sem apontar ações. Eventos desse tipo só servem para o Currículo Lattes e queimar dinheiro em final de administração", o que reforça a necessidade de maior participação popular em diversas atividades. Também foi apontado por alguns respondentes que os temas eram muito genéricos, e que, como continuidade ao evento, seria interessante que ações ocorressem com direcionamentos mais específicos.

Observando o número de ações por GT e por tópico apresentado, pode-se analisar que (i) o número de ações por GT teve uma grande variação; (ii) ao observar as ações individualmente, observa-se que o GT4 foi o que mais apresentou ações pontuais, e uma provável explicação para isso é o perfil das pessoas que participaram do GT4 (diversos participantes que atuam diretamente nos problemas relacionados a mobilidade); (iii) o GT que mais apresentou ações rotuladas por TIC foi o GT4, e a provável explicação também é a mesma do item anterior; e (iv) as TICs podem ser utilizadas também para auxiliar a comunicação entre governo e população, utilizadas em outros trabalhos apresentados na revisão da literatura (Seção 4). A fim de sumarizar os grandes tópicos que deve ser tratados a partir das ações apontadas pelos grupos de trabalho, cada uma das ações foi enumerada para cada GT. Os tópicos são apresentados na primeira coluna da Tabela 1. Ainda nessa tabela, nas colunas dois a quatro são apresentados o número de ações rotuladas por GT (GT1 a GT4) e, entre parênteses, o índice numérico de cada uma das ações, conforme foram rotuladas. Na última coluna, é apresentado o total de ações rotuladas com cada um dos tópicos. Algumas observações importantes no sumário apresentado nessa tabela: (i) educação e comunicação entre governo e população foram dois tópicos apontados por todos os GTs; (ii) era esperado que o GT2, que discutia questões diretamente ligadas ao cidadão, apontassem ações para transparência e participação popular, mas foi interessante observar que também foi apontada pelo GT1 (economia).

\section{Conclusões e Trabalhos Futuros}

Neste trabalho, é apresentado um estudo de caso para acesso à opinião dos cidadãos de Rio das Ostras (RJ) no processo de construção de um projeto para implementação de 
Tabela 1 - Tópicos utilizados para rotular as ações dos GTs

\begin{tabular}{l||c|c|c|c||c}
\multicolumn{1}{l||}{ Tópicos } & GT1 & GT2 & GT3 & GT4 & Total \\
\hline \hline educação & $1(1)$ & $1(6)$ & $1(7)$ & $1(4)$ & 4 \\
\hline $\begin{array}{l}\text { comunicação entre governo e } \\
\text { população }\end{array}$ & $4(2,5,6,8)$ & $2(3,8)$ & $3(1,5,10)$ & $1(16)$ & 10 \\
\hline \begin{tabular}{l|c} 
inovação \\
transparência e participação \\
popular
\end{tabular} & $2(3,7)$ & - & $3(2,4,6)$ & - & 5 \\
\hline $\begin{array}{l}\text { cultura } \\
\text { TIC }\end{array}$ & - & $\begin{array}{c}1 \\
(4)\end{array}$ & $\begin{array}{c}3 \\
(4,5,7)\end{array}$ & $\begin{array}{c}1 \\
(3)\end{array}$ & 2 \\
\hline governo executivo e legislativo & - & - & $3(9,11,12)$ & $9(2,5,6,9-14)$ & 12 \\
\hline
\end{tabular}

soluções em Cidades Inteligentes. É apresentado o método utilizado para coleta das opiniões, bem como uma análise das opiniões e ideias sugeridas. Pode-se concluir que o método permitiu coletar a sugestão de diversas ações por parte da população. É importante observar que os itens "transparência" e "uso de TICs" foram abordados pelos diversos Grupos de Trabalho (GTs) do III ENCIC - o GT de Economia apontou como ações a criação de canais de comunicação eficientes para interagir com o cidadão, para amenizar a falta de comunicação e criação de um portal de transparência do município para tornar o acesso às informações eficiente e eficaz; e o GT que tratou assuntos ligados à questão humana apontou que o projeto deve ser iniciado com ações para transparência, com o governo e a governança como eixo central do projeto. A importância do evento realizado está em considerar o papel subjetivo de cada parte interessada na cidade, já que a cidade não é inteligente por si mesma, mas sim criandose valor público para os cidadãos. Ainda, os resultados obtidos com o evento também levaram em consideração que a tecnologia é um aspecto central da CI, usada no serviço das iniciativas inteligentes para a qualidade de vida na cidade. Ambas as questões, apontadas por [COCCHIA 2014], foram observadas nas ações apontadas pelos GTs.

No entanto, é importante observar que o número de pessoas que participaram no processo foi bastante reduzido, se comparado ao tamanho total da população do município (mais de cem mil habitantes). Ainda assim, todos os grupos apontaram ações para aumentar a comunicação entre governo e população. Isso fortalece a necessidade de ações que vão ao encontro da transparência pública de maneira ativa por parte do governo, que pode ser realizada por meio de ações de governo eletrônico. Esse é o primeiro degrau de transparência que deve ser galgado pelo município, segundo [ALÓ 2009]. No processo realizado para coleta das opiniões, não foi realizado um levantamento mais amplo do perfil dos participantes, considerada uma limitação deste trabalho. Essa informação pode auxiliar a identificar se há algum viés ou relação entre o perfil dos participantes e as ideias sugeridas. Como trabalhos futuros, é necessário avaliar a qualidade das ideias sugeridas em cada um dos GTs, bem como avaliar a rotulação das ideias por tópicos com outras pessoas. Também, é necessário ampliar o acesso à população para aumentar a coleta de opiniões da população, que pode ser realizada tanto pela web quanto por outros encontros presenciais. 


\section{Agradecimentos}

A autora agradece à SECTI de Rio das Ostras, pelo fornecimento dos dados para o estudo de caso apresentado; e aos avaliadores do evento, por apontar pontos importantes para a melhoria deste trabalho.

\section{Referências}

Aló, C.C. (2009). Uma Abordagem para Transparência em Processos Organizacionais. Tese de Doutorado, PUC-Rio, Brasil.

Bakici, T.; Almirall, E.; Wareham, J. (2013) A Smart City Initiative: the Case of Barcelona. J. Knowledge in Economics, vol. 4, p. 135-148.

Carayannis, E. G.; Campbell, D. F. J. (2009). 'Mode 3' and 'Quadruple Helix': toward a 21 st century fractal innovation ecosystem. International Journal of Technology Management, vol. 46, n. 3, p. 201-234.

Cocchia, A (2014). Smart and Digital City: A Systematic Literature Review. Chapter 2. In: DAMERI; ROSENTHAL-SABROUX, 2014.

Dameri, R. P.; Rosenthal-Sabroux, C. (2014). Smart City and Value Creation. Chapter 1. In: DAMERI; ROSENTHAL-SABROUX, 2014.

Dameri, R. P.; Rosenthal-Sabroux, C. (eds.) (2014). Smart City: How to Create Public and Economic Value with High Technology in Urban Space, Springer Verlag.

European Commission. (2011) A renewed EU strategy 2011-14 for Corporate Social Responsibility. Disponível em: ec.europa.eu/social/BlobServlet?docId=9470. Acessado em 30/03/2017.

Giffinger, R.; Gudrun, H. (2010) Smart cities ranking: an effective instrument for the positioning of cities? ACE: Architecture, City \& Environ., vol. 4, n. 12, p. 7-25.

Lombardi, P. et al. (2011) An Advanced Triple-Helix Network Model for Smart Cities Performance. Green and Ecological Technologies for Urban Planning: Creating Smart Cities. IGI Global, p. 59-73.

Mechant, P. et al. (2012) E-deliberation 2.0 for smart cities: a critical assessment of two 'idea generation' cases. Int. J. Electronic Governance, vol. 5, n. 1, 2012.

Schuurman, D. et al (2012) Smart Ideas for Smart Cities: Investigating Crowdsourcing for Generating and Selecting Ideas for ICT Innovation in a City Context. Journal of Theoretical and Applied Electronic Commerce Research, vol. 7, n. 3, p. 49-62.

Silva, S.B.; Bittencourt, C.C. (2015) Living Labs: Rumo a um Quadro Conceitual. In: XVI Congresso Latino-Iberoamericano de Gestão da Tecnologia - ALTEC 2015.

Terán, L.; Kashina, A.; Meier, A. (2016) Maturity Model for Cognitive Cities: Three Case Studies. Chapter 3. In: PORTMANE, E.; FINGER, M. (eds.). Towards Cognitive Cities: Advances in Cognitive Computing and its Application to the Governance of Large Urban Systems 